\title{
Randomised trial of elective stenting after successful percutaneous transluminal coronary angioplasty of occluded coronary arteries
}

\author{
J Hancock, M R Thomas, S Holmberg, R J Wainwright, D E Jewitt
}

\begin{abstract}
Background-The value of angioplasty in occluded coronary arteries is limited by a restenosis/reocclusion rate of $50-70 \%$. In patients with subtotal occlusion, stent implantation has been shown to reduce clinical and angiographic restenosis. Retrospective observational studies have suggested that stenting could reduce restenosis in total occlusions. The value of sustained coronary patency on global and regional left ventricular function in this clinical setting has not been defined clearly.
\end{abstract}

Objectives-To assess the medium term effect of elective intracoronary stent deployment after successful percutaneous transluminal coronary angioplasty (PTCA) of an occluded coronary artery. Methods-Sixty patients with a total coronary occlusion successfully treated by PTCA were randomised to receive an intracoronary stent or no stent. Patients underwent clinical and angiographic follow up at six months.

Results-Thirty patients received a stent (group A) and 30 were treated by angioplasty alone (group B), all with initial success. One patient in group $B$ required repeat angioplasty with stenting at 24 hours and one patient died after 10 days. Angiographic follow up was available for 57 patients. This showed a significantly reduced reocclusion rate in group $A$ compared with group B $(7 \% v 29 \%$, p $<0.01)$ and a tendency to a reduced restenosis rate $(22 \% v 40 \%, p=0.105)$ in patients with no reocclusion. Left ventricular function, both global and regional, improved in group A. Only the regional left ventricular function in the area supplied by the target coronary artery improved in group B. Recurrence of symptoms and clinical events such as repeat angioplasty, coronary artery bypass grafting, death or myocardial infarction tended to be reduced in group A (4 (13\%) $v 9(30 \%))$.

Conclusions-Intracoronary stent insertion is effective in reducing the rate of reocclusion and shows a trend towards reduced restenosis after opening of a total coronary occlusion by balloon angioplasty. Sustained patency of the target coronary artery is associated with improvement in global and regional left ventricular function.

(Heart 1998;79:18-23)
Keywords: intracoronary stenting; total coronary occlusion; left ventricular function

Percutaneous transluminal coronary angioplasty (PTCA) in patients with chronic total occlusion (defined as total interruption of the vessel with thrombolysis in myocardial infarction (TIMI) grade 0 or $1^{1}$ flow for at least three days $^{2}$ ), has been associated with an initial primary success rate of $50-75 \%$ and a high restenosis rate of the order of $50-70 \% .^{3}$ Restenosis/reocclusion rates may be more favourable for TIMI 1 type lesions than TIMI 0 . Initial success rates may be higher for more recent occlusions but the reocclusion/ restenosis rates remain high. ${ }^{4}$ Because of this, operators have lacked enthusiasm for this technique and have favoured coronary artery bypass grafting for the treatment of totally occluded coronary arteries.

More recently, a retrospective analysis of intracoronary stent implantation after balloon angioplasty of chronic total occlusion has suggested that this would lead to a reduced restenosis/reocclusion rate. $^{2}$

Additionally, sustained patency of infarct related coronary arteries successfully opened with balloon angioplasty either early ${ }^{5}$ or up to nine months after myocardial infarction has been shown to cause improvement in global and regional left ventricular function, at least if there is normal flow (TIMI 3). ${ }^{48}$

The recently published Stenting in Chronic Coronary Occlusion (SICCO) study is the only randomised trial of stenting in this clinical setting. ${ }^{9}$ This showed a reduced restenosis rate in the stent group but no significant difference in reocclusion rate. No assessment of left ventricular function was made.

Therefore, we have performed a prospective randomised trial of elective coronary stenting after successful balloon angioplasty of a total coronary occlusion; the objective being to assess further the restenosis/reocclusion rate and in addition record the changes in global and regional left ventricular function.

\section{Methods}

PATIENTS

From May 1994 to October 1995, patients who presented with ischaemic cardiac chest pain and a total coronary occlusion were considered for randomisation. Only patients with complete obstruction of the vessel with TIMI grade 0 or 1 flow that was at least three days old were considered eligible for randomisation. 
During the period May 1994 to October 1995 the angioplasty registry recorded attempted total occlusion angioplasty in 187 patients. Only patients who had an occluded artery at the beginning of the procedure could be considered - that is, procedural stent occlusions were not included. The occlusion was successfully crossed in 157 (84\%). After balloon dilatation distal flow was poor in 21 patients. In one further patient the wire was felt not to be in the true lumen leaving a total of $135(72 \%)$. Forty four were excluded. Of these, 13 had stent thrombosis, eight were in coronary vein grafts, eight were for acute myocardial infarction, eight had angiographic thrombus, six had vessels that were less than $3 \mathrm{~mm}$ in diameter, and one had femoral trauma so that a stent had to be avoided because of the anticoagulation regimen. Of the 91 remaining, $60(66 \%)$ were randomised to the study (table 1 ). The patients randomised, therefore, were a selected group of the total patients undergoing PTCA of a coronary occlusion at this institution. Thirty one patients eligible for randomisation were not included. The reasons for this were multifactorial, but include consent not received, operator inexperience with stents, contraindication to anticoagulation (and therefore stenting), and other unknown factors. No patients were deliberately excluded.

The occluded artery (the target vessel) was the left anterior descending coronary artery in 28 patients, the right coronary artery in 28 patients, and a dominant circumflex artery in four patients. Most patients (57) underwent single vessel PTCA, three patients had two vessel PTCA. Forty two patients had TIMI 0 flow (70\%), 18 patients had TIMI 1 flow $(30 \%)$. Timing of the occlusion was estimated clinically. Any occlusions that were thought to be less than three days old were excluded, though 19 patients had experienced a recent (3-14 days) myocardial infarction.

The patient group consisted of 38 men and 22 women with a mean age of 60 years (range 36-79). All patients signed informed consent including six month follow up angiography. Clinical follow up occurred at the same time as the six month follow up angiogram.

\section{STUDY DESIGN}

The target vessel was reopened with standard balloon techniques. Only after successful balloon angioplasty had been performed and the operator was happy with the final result were the patients randomised to receive a stent or no stent. Successful initial PTCA was defined as

Table 1 Patient demographic data

\begin{tabular}{lrr}
\hline & Group A & Group B \\
\hline Mean age (years) & 61 & 60 \\
Number of men (\%) & $16(53)$ & $22(73)$ \\
Mean (SD) time of occlusion (weeks) & $14(17)$ & $9(10)$ \\
Recent MI (3-14 days) (\%) & $8(27)$ & $11(37)$ \\
TIMI 0 flow (\%) & $21(70)$ & $21(70)$ \\
Target coronary artery & $14(47)$ & $14(47)$ \\
$\quad$ Left anterior descending (\%) & $1(3)$ & $3(10)$ \\
Circumflex (\%) & $15(50)$ & $13(43)$ \\
Right (\%) & $23(77)$ & $21(70)$ \\
Retrograde filling (\%) & & \\
\hline
\end{tabular}

less than $50 \%$ residual stenosis (visual assessment) with distal TIMI grade 3 flow.

In the group of patients randomised to receive a stent (group A), an attempt was made to use only a single Palmaz Schatz stent at the site of the original occlusion, though down stream ballooning was frequently performed. This was done intentionally both to limit cost as well as the length of the stented segment, as we believe increased stent length may increase subsequent restenosis. In addition, our principal aim in deploying the stent was to limit reocclusion, which usually occurs at the original site of occlusion. The stents were deployed optimally with a high pressure balloon inflation. In the group of patients who did not receive a stent (group B), a satisfactory balloon angioplasty result was achieved before randomisation. No further balloon inflations were performed in this group after randomisation. There was no significant difference in the mean time (SD) of occlusion between the two groups (14 (17) weeks in group A and 9 (10) weeks in group B). After occlusion angioplasty with or without stent implantation, the patients received a standard anticoagulation regimen. Group A received prolonged heparin maintaining an activated clotting time (ACT) of 150-200 until the international normalised ratio (INR) was greater than $2.0,300 \mathrm{mg}$ of aspirin a day, and anticoagulation with warfarin for one month. Group B received the standard regimen of 1000 units per hour of heparin overnight and $300 \mathrm{mg}$ of aspirin a day.

\section{Quantitative angiography}

Quantitative coronary angiography was retrospectively performed by two investigators using the guiding catheter as a scaling device. Calipers were used to measure the reference lumen diameter and the final minimal luminal diameter. The occlusion length was not measured because of the difficulty in estimating this accurately. The reference luminal diameter was taken to be the average diameter (in two orthogonal views) of a normal appearing segment proximal to the occlusion. In assessing the minimal luminal diameter, two orthogonal views were used and the smallest selected for measurements. The follow up measurements were taken in the same projection. Follow up percentage stenosis was then calculated, as well as relative loss and overall gain. Angiographic restenosis was defined as greater than or equal to $50 \%$ stenosis relative to the proximal reference diameter on follow up angiography.

\section{Left ventricular function}

Left ventricular function, both global and regional, was assessed before intervention and at six month follow up. Global left ventricular function was assessed angiographically by tracing the end diastolic and end systolic perimeters in the $30^{\circ} \mathrm{RAO}$ projection and calculating ejection fraction by the area length method. Only this view was used as the occluded (target) coronary artery was either the left anterior descending coronary artery, the right coronary artery or a dominant circumflex artery in all cases. Regional left ventricular 
Table 2 Procedural and six month angiographic results

\begin{tabular}{llll}
\hline & Group A (stent) & Group B (no stent) & p value \\
\hline Procedural result & & & \\
$\quad$ Reference diameter & $3.3 \mathrm{~mm}$ & $3.5 \mathrm{~mm}$ & $\mathrm{NS}$ \\
Minimal luminal diameter & $3.3 \mathrm{~mm}$ & $2.8 \mathrm{~mm}$ & $<0.001$ \\
Residual stenosis & $-1.4 \%$ & $20.3 \%$ & $<0.0001$ \\
Six month follow up & $\mathrm{n}=29$ & $\mathrm{n}=28$ & \\
Reference diameter & $3.3 \mathrm{~mm}$ & $3.5 \mathrm{~mm}$ & $\mathrm{NS}$ \\
Minimal luminal diameter & $2.0 \mathrm{~mm}$ & $1.5 \mathrm{~mm}$ & $<0.05$ \\
Residual stenosis & $35.4 \%$ & $58.7 \%$ & $<0.01$ \\
Relative loss & $0.41 \mathrm{~mm}$ & $0.38 \mathrm{~mm}$ & $\mathrm{NS}$ \\
Overall gain & $0.65 \mathrm{~mm}$ & $0.41 \mathrm{~mm}$ & $<0.01$ \\
Reocclusion & $2 / 29(7 \%)$ & $8 / 28(29 \%)$ & $<0.02$ \\
Restenosis (without reocclusion) & $6 / 27(22 \%)$ & $8 / 20(40 \%)$ & $\mathrm{NS}$ \\
Restenosis/reocclusion & $8(28 \%)$ & $16(57 \%)$ & $<0.01$ \\
\hline
\end{tabular}

function was assessed using the centreline chordal shortening method in which a computer generated line is drawn through the midpoint of the end diastolic and end systolic left ventricular perimeters. The ventricle is then divided into 100 chords perpendicular to this line and the motion of each chord relative to the line measured. For the purposes of this study chords $10-60$ were taken to represent the left anterior descending coronary artery and chords 60-90 the right coronary artery. The motion of the chords is normalised for heart size by dividing by the end diastolic perimeter. ${ }^{9}$ To establish normal values for chordal shortening 10 patients matched for age, sex, and drug history, who had normal coronary anatomy and left ventricular function were analysed. Chordal shortening in this group of patients was found to be 5.1 in the 20 right and left coronary beds assessed.

\section{End points}

The primary end point was angiographic reocclusion. Secondary end points include six month angiographic minimal luminal diameter and a combined six month clinical event rate consisting of repeat PTCA, coronary artery bypass grafting, and myocardial infarction.

\section{Statistics}

Quantitative angiographic parameters were compared using a separate variance $t$ test. Left ventricular ejection fraction and regional chordal shortening were compared using a paired $t$ test. Fisher's exact test was used to compare categoric variables such as reocclusion and incidence of angiographic restenosis.

\section{Results}

PROCEDURAL RESULTS

Of the 30 patients randomised to group A, 26 patients received a single $15 \mathrm{~mm}$ Palmaz Schatz stent, one patient received an $18 \mathrm{~mm}$ Palmaz Schatz stent, one patient had two Palmaz Schatz stents inserted, and of two patients with tortuous anatomy one received a Medtronic (Wiktor) stent while one received two AVE stents. In one patient, the stent was delivered proximal to the occlusion because it was not possible to negotiate the stent to the site of occlusion and in one patient, a stent was "dropped" in the descending aorta with no clinical sequelae. A second stent was deployed with good result. All patients were analysed on an "intention to treat" basis until a clinical end point was reached.

\section{IN-HOSPITAL EVENTS}

There was no procedural crossover caused by obstructive dissection in group B. The length of the inpatient stay was 5.4 days for group A and 2.3 days for group B. There was no clinical or angiographic reocclusion but there was one patient in group B who had post angioplasty chest pain with electrocardiographic changes at 24 hours. The angiogram showed a dissection with a stenosis of $>70 \%$ so the patient received a stent as a clinical end point. Creatinine phosphokinase was not routinely measured after procedure. The absence or occurrence of non-Q wave myocardial infarction was estimated on the basis of chest pain with electrocardiographic changes. There was one significant femoral haematoma requiring transfusion in group A. There were no inpatient deaths or referrals for coronary artery bypass surgery.

SIX MONTH ANGIOGRAPHIC FOLLOW UP

Six month angiographic follow up was available for 57 patients. Table 2 shows the results.

Two patients declined six month angiography, one in group A and one in group B and both were clinically asymptomatic. One death occurred 10 days after procedure in group B and postmortem examination indicated reocclusion of the left anterior descending coronary artery.

\section{LEFT VENTRICULAR FUNCTION}

Left ventricular angiograms before intervention and at six month follow up were available for 52 patients. Global left ventricular ejection fraction improved from $59.5 \%$ to $71.1 \%$ in group A ( $p<0.001)$ and from $54.5 \%$ to $62.2 \%$ in group B (NS) (fig 1). Initial left ventricular function was not significantly different in the two groups, but there was a greater improvement in ejection fraction at six months in group A compared with group B $(\mathrm{p}<0.02)$.

Regional left ventricular function was assessed for the target coronary bed as well as the contralateral coronary bed such that if the target artery was the left anterior descending coronary artery the target bed refers to the area of myocardium supplied by this coronary artery and the contralateral bed to the area supplied by the right coronary artery and vice versa.

Considering first the target coronary bed, chordal shortening improved in this region

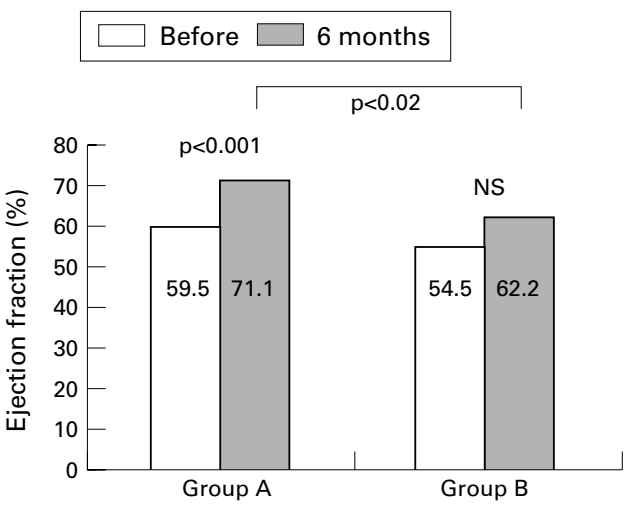

Figure 1 Global left ventricular function. 


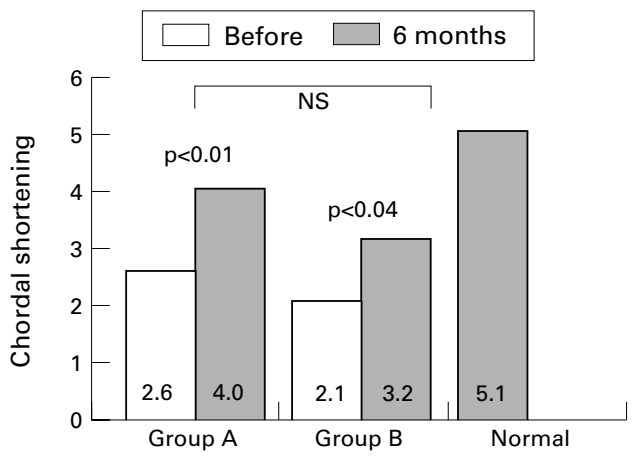

Figure 2 Regional left ventricular function - target coronary bed.

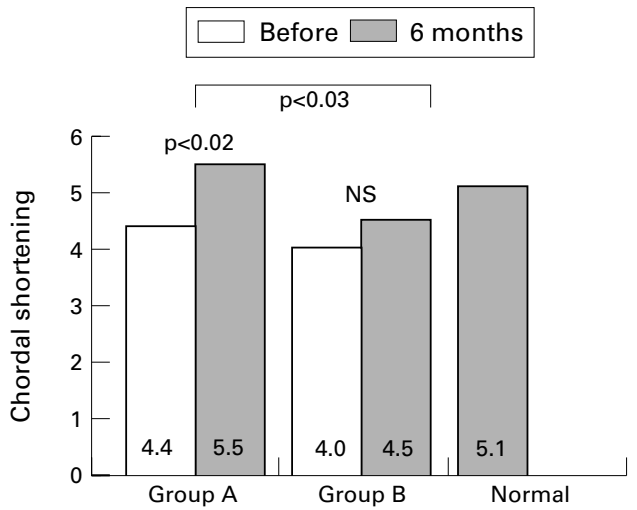

Figure 3 Regional left ventricular function - contralateral coronary bed.

from 2.6 to 4 in group A $(\mathrm{p}<0.01)$. There was a similar improvement in group B (2.1 to 3.2 $\mathrm{p}<0.04$ ) (fig 2). Considering the contralateral coronary bed, chordal shortening improved significantly in this region in group A (4.4 to $5.5 \mathrm{p}<0.02)$ but not in group B (4 to $4.5 \mathrm{NS}$ ) (fig 3). The improvement in the contralateral bed in group A was significantly greater than group $B$ at six months $(\mathrm{p}<0.03)$.

CLINICAL EVENTS

Clinical follow up of all patients revealed four clinical events in group A and nine in group B $(13 \% v 30 \%, \mathrm{p}=0.105)$ (fig 4$)$. Three patients had repeat PTCA and one had coronary artery bypass grafting during the follow up period in

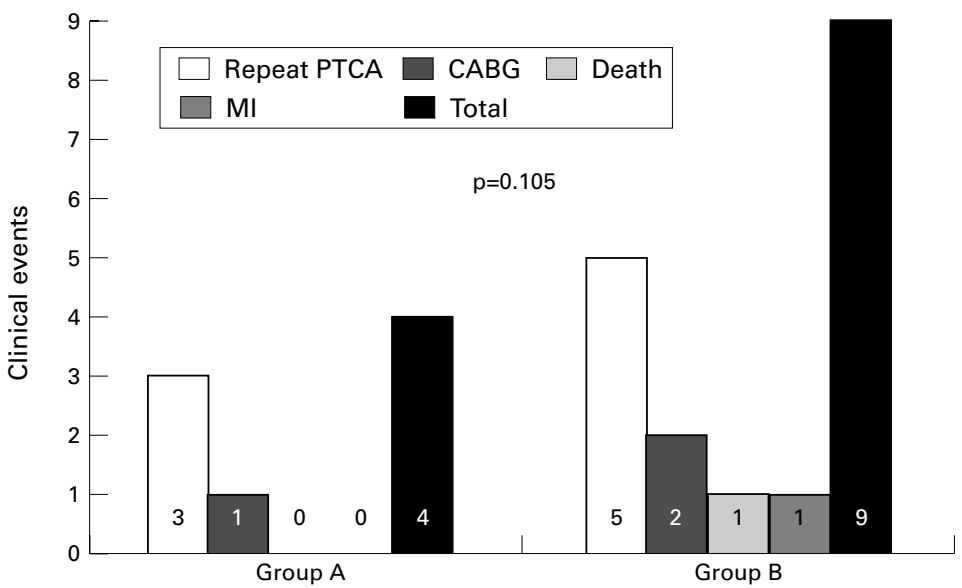

Figure 4 Clinical events. CABG, coronary artery bypass grafting; $M I$, myocardial infarction. group A. Five patients had repeat PTCA, two had coronary artery bypass grafting, one died, and one had myocardial infarction in group $B$.

\section{Discussion}

Percutaneous revascularisation has been less successful in treating patients with total coronary occlusion than subtotal stenosis. This is because there is a lower initial success rate and subsequent high reocclusion and restenosis rates. Approximately $73-83 \%$ of these lesions can be reopened with angioplasty by experienced operators ${ }^{11-13}$ and long term benefit is limited by a restenosis rate of $50-70 \%$, which is associated with an increased clinical event rate. ${ }^{13}{ }^{14}$ Ten to 20 per cent of patients who would otherwise be suitable for angioplasty have a total coronary occlusion. ${ }^{15} 16$ Many have been referred for coronary artery bypass surgery, even in the presence of single vessel disease, because of the poor results of angioplasty in this condition. It seems probable that the use of intracoronary stent implantation may lead to a significant reduction in the restenosis/reocclusion rate compared with that for balloon angioplasty. This should result in a reduced clinical event rate. Restoration and maintenance of coronary patency with TIMI 3 flow should lead to an improvement in global and regional left ventricular function. ${ }^{12}{ }^{14-17}$

In the randomised study reported here of stenting versus balloon angioplasty alone in the treatment of chronic total occlusions, in which 30 patients received a stent and 30 were treated with balloon angioplasty alone, clinical and angiographic follow up at six months was used to evaluate angiographic restenosis and left ventricular function. Angiographic follow up was available in $95 \%$ of patients and clinical follow up in all. Angiographic reocclusion had occurred in two patients in group A (7\%) and eight $(29 \%)$ patients in group B. Angiographic restenosis on the basis of a minimal lumen diameter of $<50 \%$ of the reference diameter had occurred in six of $27(22 \%)$ of group A and eight of $20(40 \%)$ of group B (of those patients without reocclusion). The overall restenosis/ reocclusion rate was $28 \%$ in group A and $57 \%$ in group $B(p<0.01)$. Clinical events occurred in only four $(13 \%)$ patients in group $A$ and nine $(30 \%)$ in group B.

Left ventricular angiograms pre-intervention and at six month follow up were available for 52 patients $(87 \%)$. Global and regional left ventricular function improved significantly in group A, even in the contralateral myocardial bed. Only regional left ventricular function in the area of the target myocardial bed improved significantly in group B.

REASONS FOR REOCCLUSION/RESTENOSIS

Several factors were evaluated as possible risk factors for restenosis. Older occlusions, the presence of TIMI 0 compared with TIMI 1 flow, significant dissection, length of lesion, target coronary artery, final minimal lumen diameter, final per cent diameter stenosis, diabetes, and smoking history were all considered. Only final minimal lumen diameter was shown to be a significant predictor of follow up 
minimal lumen diameter. This may be because the number of patients in each group is too small for extensive subgroup analysis. In elective stenting after balloon angioplasty of coronary occlusions a possible link between the presence of moderate to severe dissection and the follow up minimal lumen diameter has been suggested. ${ }^{2}{ }^{18}$

REASONS FOR BENEFIT

In group $\mathrm{A}$ the final minimal lumen diameter was significantly greater than in group B, which may in part explain the reduced rate of reocclusion/restenosis in this group, particularly as there was no significant difference in the relative loss between the two groups at six month follow up, but there was a significant difference in relative gain $(\mathrm{p}<0.01)$. Previous investigators have found a significant relation between acute gain and larger final minimal lumen diameter achieved with stent implantation, compared with balloon angioplasty alone, in subtotally stenosed lesions. ${ }^{18}$ Stents may limit dissection induced reclosure. It has been suggested that a dissection related reocclusion after balloon angioplasty might occur very soon after the procedure yet not present clinically because of previously established collateral flow, falsely increasing the restenosis/ reocclusion rate, when the mechanism was early abrupt closure. ${ }^{2}$ Although the numbers in this study are too small to be certain, however, two patients reoccluded early (one died and one presented with a myocardial infarction in group B) and one patient developed chest pain and repeat angiography showed a stenosing dissection, which was then stented, which would dispute the above theory. Most reocclusions were asymptomatic (nine, $82 \%$ ), which may actually represent a gradual restenotic process with development of further collaterals and eventual reocclusion rather than abrupt reclosure. This is particularly probable because most patients had retrograde filling of the occluded coronary artery before intervention, implying pre-existing collateral flow, yet all presented with chest pain. If the reocclusive process was abrupt reclosure leading to the reopening of pre-existing collaterals then an acute recurrence of preintervention symptoms might be expected.

IMPROVEMENTS IN LEFT VENTRICULAR FUNCTION Left ventricular function is the most important predictor of late survival in patients with coronary artery disease. Early restoration of coronary artery patency and the persistence of patency are important goals in thrombolytic treatment. The mechanism by which thrombolytic treatment improves long term survival is the preservation of left ventricular function. ${ }^{19} 20$ Previous studies have shown that the earlier reperfusion occurs the greater the myocardial salvage, less left ventricular dilatation occurs, and mortality is reduced. ${ }^{5}$ Only complete reperfusion defined as TIMI 3 flow in the infarct related artery confers this benefit, and residual stenosis has been shown to be an important predictor of changes in left ventricular volume in the first year after myocardial infarction. ${ }^{21} \mathrm{~A}$ clear benefit of early routine angioplasty of the infarct related artery early after thrombolysis has not been shown. Less is known about the preservation of left ventricular function after late restoration of optimal perfusion, in the chronic phase after myocardial infarction, though improvements in left ventricular function can occur even with restoration of normal (TIMI 3) perfusion as late as nine months after myocardial infarction. ${ }^{8}$ Some investigators suggest that these improvements occur without any increase in left ventricular end diastolic volume. However, in the presence of reduced flow or reocclusion, improvements in left ventricular function can occur but this tends to be because of increased left ventricular end diastolic volume implying a remodelling process. $^{22}$

In this study global left ventricular function improved significantly only in group A. This was probably because of the lower reocclusion rate in this group, as the restenosis rate was similar in both groups and all patent arteries had TIMI 3 flow. This is largely in agreement with previous studies. The regional left ventricular function improved significantly in both groups in the target myocardial bed despite the higher reocclusion rate in group B. This may result from the process of reocclusion being one of gradual restenosis rather than abrupt reocclusion permitting improved collateral formation and preventing remodelling. On the other hand, regional function in the contralateral myocardial bed improved significantly only in group A, which implies normal perfusion is required for this effect. The mechanism for this is speculative, but it may reflect tethering of normal myocardial segments by dysfunctional segments in the target myocardial bed, which are restored to normal function after restoration of persistent normal perfusion.

\section{LIMITATIONS OF THE STUDY}

The small numbers in this study have limited subgroup analysis and therefore precluded identification of the mechanism of benefit on restenosis rate after intracoronary stent insertion following angioplasty of total chronic occlusions. Quantitative angiography was performed with digital calipers by two unblinded independent observers instead of computerised quantitative angiography with automatic edge detection. It is possible that this may have introduced bias particularly when estimating restenosis in the two groups.

The small subgroup numbers may have adversely effected the analysis of left ventricular function. No assessment of myocardial viability was performed, though all patients presented with chest pain and most $(95 \%)$ had single vessel disease implying ischaemia in the myocardial bed supplied by the occluded coronary artery.

FUTURE DIRECTIONS

Further assessment of long term outcome in terms of morbidity and mortality is necessary, particularly in relation to target coronary artery flow and left ventricular function. It is possible that asymptomatic patients with an occluded 
coronary artery and impaired left ventricular function may benefit from restoration of coronary flow with stenting to improve perfusion long term. A study of such a group of patients with inclusion of a viability assessment should be undertaken.

CONCLUSIONS

This study confirms the recently published stenting in chronic coronary occlusion (SICCO) study $^{9}$ and adds assessment of changes in global and regional function. The SICCO study is the only other randomised trial of stenting in this clinical setting and indicated a reduced restenosis rate in the stent group $(32 \% v 74 \%, \mathrm{p}<0.001)$ and a strong trend to reduced reocclusion of the target artery $(12 \% v$ $26 \%, p=0.058)$. The failure of reocclusion to reach statistical significance seems to reflect the comparatively high stent thrombosis rate $(6.9 \%)$, which was not seen in this study. The SICCO study also indicated a reduced target lesion revascularisation rate at 300 days $(22 \%$ v $42 \%, \mathrm{p}=0.025$ ).

In our study the overall restenosis/ reocclusion rate was reduced from $57 \%$ to $28 \%$ $(\mathrm{p}<0.01)$ by use of an intracoronary stent. Reocclusion rates were reduced from $29 \%$ to $7 \%(\mathrm{p}<0.02)$. Recurrence of symptoms and further clinical events tended to be reduced in the stent group.

Intracoronary stent insertion is therefore effective in reducing the rate of reocclusion and restenosis after opening of a total coronary occlusion by balloon angioplasty. Importantly, this study shows that stents have this benefit even when a satisfactory balloon angioplasty result has been obtained.

Increasing data support the elective deployment of a coronary stent after successful balloon angioplasty of an occluded coronary artery. This strategy is justified to maintain coronary patency and reduce restenosis, resulting in improved left ventricular function and a reduced clinical event rate.

1 TIMI Study Group. The thrombolysis in myocardial infarction (TIMI) trial: phase I findings. $N$ Engl $\mathcal{f} \mathrm{Med}$ 1985;312:932-6.

2 Goldberg SL, Colombo A, Maiello L, Borrione M, Finci L, Almagor Y. Intracoronary stent insertion after balloon Almagor Y. Intracoronary stent insertion after balloon angioplasty of ch

3 Meier B. Total coronary occlusion: a different animal? f Am Coll Cardiol 1991;17:50-7B.

4 Dzavik V, Beanlands DS, Davies RF, Leddy D, Marquis JF, Teo KK, et al. Effects of late percutaneous translumina coronary angioplasty of an occluded infarct-related coronary artery on left ventricular function in patients with a recent $(<6$ weeks) Q-wave acute myocardial infarction (total occlusion post-myocardial infarction intervention tudy (TOMIIS) - a pilot study. Am f Cardiol 1994;73: 856-61.

5 Gruppo Italiano per lo Studio della Streptochinasi nell'Infarcto miocardico (GISSI). Effectiveness of intravenous thrombolytic treatment in acute myocardial infarction. Lancet 1986;i:397-401.

6 Second International study of Infarct Survival Collaberative Group. Randomised trial of intravenous streptokinase, oral aspirin, both, or neither among 17,187 cases of suspecte
myocardial infarction: ISIS-2. Lancet 1988;ii:349-60.

7 Miketic S, Carlsson J, Tebbe U. Improvement of global and regional left ventricular function by percutaneous transluminal coronary angioplasty after myocardial infarction. $\mathcal{F}$ Am Coll Cardiol 1995;25:843-7.

8 Linderer T, Guhl B, Spilberg C, Wunderlich W, Schnitzer L, Schroeder R. Effect on global and regional left ventricular function by percutaneous transluminal coronary angioplasty in the chronic stage after myocardial infarction. Am f Cardiol 1992;69:997-1002.

9 Sirnes PA, Golf S, Myreng Y, Molstad P, Emanuelsson H, Albertson $\mathrm{P}$, et al. Stenting in chronic coronary occlusion (SICCO): a randomised, controlled trial of adding stent implantation after successful angioplasty. $\mathcal{F} \mathrm{Am}$ Coll Cardiol 1996;28:1444-51.

10 Sheehan FH, Bolson EL, Dodge HT, Mathey DG, Schofer $\mathrm{J}$, et al. Advantages and applications of the centreline met et al. Advantages and applications of the centreline Circulation 1986;74:293-305.

11 Maiello L, Colombo A, Gianrossi R, Mutinelli MR, Bouzon $\mathrm{R}$, Thomas $\mathrm{J}$, et al. Coronary angioplasty of chronic occlusions: factors predictive of procedural success. $\mathrm{Am}$ Heart f 1992;124:581-4

12 Violaris AG, Serruys PW Total coronary occlusion: current approaches for improving acute success and long term outcome. Interventional Cardiology Monitor 1995;2:61-7.

13 Kinoshita I, Katoh O, Nariyama J, Otsuji S, Tateyama H, et al. Coronary angioplasty of chronic total occlusions with bridging collateral vessels: immediate and follow up outcome from a large single center experience. $\mathcal{F} \mathrm{Am}$ Coll Cardiol 1995;26:409-15.

14 Safian RD, McCabe C, Sipperley ME, McKay RG, Baim DS. Initial success and long term follow up of percutaneous transluminal coronary angioplasty in chronic occlusions versus conventional stenoses. Am f Cardiol 1988;61: 23-8G

15 Ruocco NA, Ring ME, Holubkov R, Jacobs AK, Detre KM, Faxon DP, et al. Results of coronary angioplasty of chronic total occlusions (the National Heart, Lung, and Blood Institute 1985-1986 Percutaneous Transluminal Angioplasty Registry.) Am f Cardiol 1992;69:69-76.

16 Bell MR, Berger PB, Bresnahan JF, Reeder GS, Bailey KR, Holmes DRJr. Initial and long term outcome of 354 patients following coronary balloon angioplasty of total coronary artery occlusions. Circulation 1992;85:1003-11.

17 Puma JA, Sketch MH, Tcheng JE, Harrington RA, Phillips HR, Stack RS, et al. Percutaneous revascularisation of chronic coronary occlusions: an overview. $\mathscr{f} \mathrm{Am}$ Coll Cardiol 1995;26:1-11.

18 Kuntz RE, Gibson CM, Nobuyoshi M, Baim DS. Generalised model of restenosis after conventional balloon angioplasty, stenting and directional atherectomy. $\mathcal{F ~} \mathrm{Am}$ Coll Cardiol 1993;21:15-25.

19 Dalen JE, Gore JM, Braunwald E, Borer J, Goldberg RJ, Passamani ER, et al. Six and twelve month follow up of the phase I thrombolysis in myocardial infarction (TIMI) trial. Am f Cardiol 1988;62:179-85.

20 Mock MB, Ringovist I, Fisher LD, Davis KB, Chaitman BR, Kouchoukos NT, and participants in The Coronary Artery Surgery Study. Survival of medically treated patients in the coronary artery surgery study (CASS) Registry. Circulation 1982;66:562-8.

21 Leung WH, Lau CP. Effects of severity of the residual stenosis of the infarct related artery on left ventricular dilatation and function after acute myocardial infarction. $7 \mathrm{Am}$ Coll Cardiol 1992;20:307-13.

22 Danchin N, Angioi M, Cador R, Tricoche O, Dibon O, Marie PY, et al. Late percutaneous recanalisation of chronic total coronary occlusion after myocardial infarction improves global and regional left ventricular function and avoids remodeling in the absence of subsequent reocclusion. Circulation 1995;92 (suppl 1):1-74. 